\title{
Angular Dependence of Universal Conductance Fluctuations in Noble-Metal Nanowires
}

\author{
E. Scheer, ${ }^{1}$ H. v. Löhneysen, ${ }^{1}$ A. D. Mirlin, ${ }^{2, *}$ P. Wölfle, ${ }^{2}$ and H. Hein ${ }^{3}$ \\ ${ }^{1}$ Physikalisches Institut, Universität Karlsruhe, D-76128 Karlsruhe, Germany \\ ${ }^{2}$ Institut für Theorie der Kondensierten Materie, Universität Karlsruhe, D-76128 Karlsruhe, Germany \\ ${ }^{3}$ Forschungszentrum Karlsruhe, Institut für Mikrostrukturtechnik, D-76021 Karlsruhe, Germany
}

(Received 17 October 1996)

\begin{abstract}
We investigate fluctuations in the magnetoconductance (measured at $T \approx 50 \mathrm{mK}$ ) of noble-metal nanowires in a mesoscopic two-lead configuration, with lengths 500 and $1000 \mathrm{~nm}$ and widths $w$ between 45 and $360 \mathrm{~nm}$. We determine the dependence of the correlation field $B_{c}$ on the angle $\theta$ between the direction of the magnetic field and the long axis of the wires. $\theta$ is changed at low $T$ continuously from $90^{\circ}$ (usual geometry) to $0^{\circ}$. We present a calculation taking into account the 3D diffusive motion of the conduction electrons describing the angular dependence of $B_{c}$. We compare this $B_{c}(\theta)$ dependence with conductance fluctuations observed while sweeping $\theta$ at constant magnetic field. [S0031-9007(97)03042-1]
\end{abstract}

PACS numbers: 73.23.-b

Universal conductance fluctuations (UCF) in metals are a direct manifestation of quantum interference of electron wave packets. They can be observed if the sample dimensions are smaller than or comparable to the phase coherence length $l_{\phi}=\left(D \tau_{\phi}\right)^{1 / 2}\left(D=v_{F} l / 3\right.$ is the diffusion constant and $\tau_{\phi}$ is the phase-breaking scattering time) for conduction electrons [1,2]. In polycrystalline metal films $l_{\phi}$ is of the order of $1 \mu \mathrm{m}$ at very low temperatures $T<1 \mathrm{~K}$, whereas the elastic mean free path $l$ is about 10 to $50 \mathrm{~nm}$. In this diffusive regime the electrical conductance $G$ is influenced by the interference of electronic trajectories which is sensitive to small changes in the Hamiltonian. $G$ is found to fluctuate around a mean value $\bar{G}$ as a function of an external control parameter as, for example, the Fermi energy [3], the transport voltage [4,5], the perpendicular magnetic field [1], configuration of the scattering centers [6] or, as we will show here, the angle between external magnetic field and current direction. In a mesoscopic two-lead configuration, i.e., when within the phase coherent sample volume $l_{\phi}^{3}$ only two measuring probes are in contact with the mesoscopic sample, the rms amplitude of the fluctuations depends only very weakly on the sample shape (length $L$, width $w$, and thickness $d$ ) as long as the transport is diffusive and coherent $\left(l \ll w, d, L \leq l_{\phi}\right)[7,8]$.

The properties of the fluctuations in all these realizations are essentially the same with the only exception being that the rms amplitude observed for fluctuations measured as a function of the magnetic field $B$ is smaller by a factor $\sqrt{2}$ because of time-reversal symmetry breaking [7]. An additional reduction of the rms amplitude by a factor of 2 occurs in metals with strong spin-orbit scattering. Altogether, one expects a saturation rms amplitude of magnetoconductance fluctuations for $\mathrm{Au}$ or $\mathrm{Ag}$ of $\mathrm{rms}$ $G_{1 D}=0.26 e^{2} / h$ for quasi-1D wires [9].

The characteristic fluctuation period $B_{c}$, the correlation field, is a measure of the area $A_{\phi}$ enclosed by typical interference paths since $A_{\phi} B_{c}=C \phi_{0}$, where $\phi_{0}=h / e$ is the elementary flux quantum, in analogy to the wellknown Aharonov-Bohm effect in ring structures [1]. Here $C$ is a constant of order unity [7]. $B_{c}$ is given by the half width at half maximum (HWHM) of the autocorrelation function $F(\Delta B)$ of the magnetoconductance curves. Because all coherent trajectories contribute to the interference pattern, $B_{c}$ should depend on the geometry of the sample if the width and/or the length of the sample become smaller than $l_{\phi}$. In the quasi-1D case (at $T=0$ ) with the magnetic field oriented perpendicular to the film plane $\left(\theta=90^{\circ}\right)$ one expects $B_{c} \approx \phi_{0} / w L$. Varying the angle $\theta$ between $B$ and the axis of the sample provides an additional possibility to change the effective transverse sample dimensions while the sample remains microscopically the same. For strictly $2 \mathrm{D}$ systems such as $2 \mathrm{D}$ electron gases complete correlation down to $\theta=10^{\circ}$ can be observed by simply scaling the $B$ axis to the projection perpendicular to the sample area $B \sin \theta$ [10], i.e., $B_{c}$ is determined by the cross section $A_{\perp}$ perpendicular to $B$, $B_{c} \propto(\sin \theta)^{-1}$.

In thin but 3D diffusive metallic films the dependence of the UCF pattern on the angle $\theta$ between current direction and magnetic field has not been explored up to now. Of course, one expects an increase of $B$ with decreasing $\theta$. As we will show in this paper $B_{c}(\theta)$ is not only determined by $A_{\perp}$, but the whole 3D sample geometry has to be taken into account. We will present a calculation based on the diffusive theory [7] that explains the unexpected angular dependence $B_{c}(\theta)$ found in our experiments.

We report measurements of the magnetoconductance at very low temperatures $T \approx 50 \mathrm{mK}$, of gold and silver wires in a two-lead configuration with wide funnel-shaped leads, with lengths $L \geq 500 \mathrm{~nm}$ and widths varying between 45 and $360 \mathrm{~nm}$. In addition, we determine for the first time the angular correlation in a fixed magnetic field by changing $\theta$ continuously. The UCF dimensionality of the narrow samples investigated here is quasi-1D in the saturated case $L, d, w \leq l_{\phi}$, whereas all 
samples are 3D with respect to the motion of the electrons between elastic scattering events [11].

The samples are produced by a standard lift-off process described elsewhere [12]. The samples were mounted inside the mixing chamber of a ${ }^{3} \mathrm{He} /{ }^{4} \mathrm{He}$ dilution refrigerator which is equipped with a 13-T superconducting solenoid. An ac-resistance bridge $(17 \mathrm{~Hz})$ records the fluctuations with a voltage resolution of about $1 \mathrm{nV}$. A mechanical feedthrough into the mixing chamber allows for tilting the sample continuously from the usual geometry with $B$ perpendicular to the film plane $\left(\theta=90^{\circ}\right)$ to a parallel alignment between $B$ and current direction while it is maintained at low temperatures. The accuracy of the angle adjustment is about $\pm 3^{\circ}$.

The experimental details and results of the measurements in the normal geometry $\theta=90^{\circ}$ have been discussed in detail previously [12,13]. The rms amplitudes of the fluctuations have been found to be in very good agreement with the theoretical predictions for the quasi1D case. Furthermore, $B_{c}$ of wires with constant $L$ and varying width $w$ shows the expected geometry dependence $B_{c} \propto \phi_{0} /(w L)$. Within our accuracy we find no systematic difference between the behavior of $\mathrm{Ag}$ or $\mathrm{Au}$ samples in their UCF properties, for both we deduce $l_{\phi} \approx 700 \mathrm{~nm}$. The contribution of the wide contact pads to the UCF is negligible for $L=1000 \mathrm{~nm}$ and leads to $B_{c} \gtrsim 400 \mathrm{mT}$ with very small amplitude for $L=500 \mathrm{~nm}$. This can clearly be distinguished from the pattern of the wires themselves $\left(B_{c} \lesssim 100 \mathrm{mT}\right)$ [13].

Figure 1 shows the magnetoconductance fluctuations in units of $e^{2} / h$ of sample 1 with $L=500 \mathrm{~nm}, w=45 \mathrm{~nm}$, and $d=25 \mathrm{~nm}$ for different angles $\theta$ in the field range

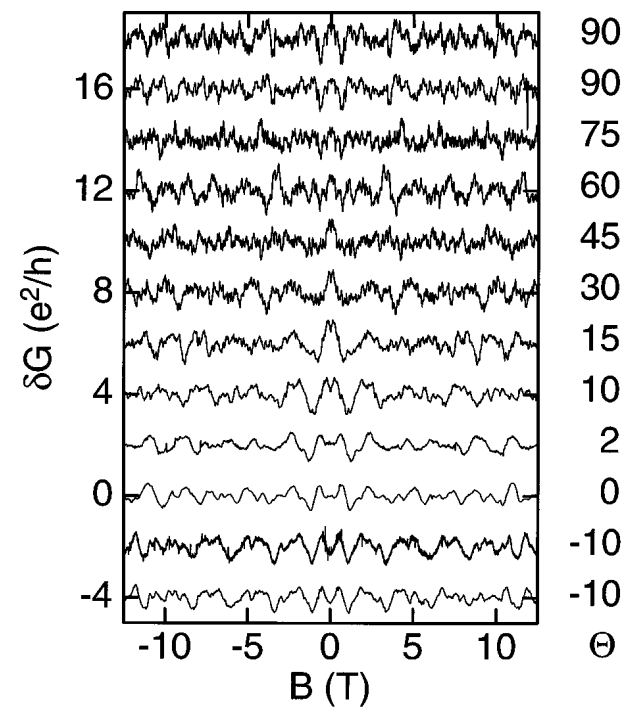

FIG. 1. Conductance fluctuations of sample 1 with $w=45$ $\mathrm{nm}, L=500 \mathrm{~nm}$, and $d=25 \mathrm{~nm}$ for various angles between magnetic field and current direction, offset by $2 e^{2} / h$ each. From top to bottom the angles are $90^{\circ}, 90^{\circ}, 75^{\circ}, 60^{\circ}, 45^{\circ}, 30^{\circ}$, $15^{\circ}, 10^{\circ}, 2^{\circ}, 0^{\circ},-10^{\circ}$, and $-10^{\circ}$. from -12.5 to $12.5 \mathrm{~T}$. The curves are offset by $2 e^{2} / h$ each for clarity. One observes that the rms amplitude remains nearly unchanged while varying $\theta$. The numerical analysis (see [12] for details) of the data yields the rms amplitudes (for $\theta=90^{\circ}$ ) given in Table I for samples 1 to 5 .

$B_{c}$ increases clearly from $\theta=90^{\circ}$ to $\theta=0^{\circ}$. This happens not only by damping of the short-period fluctuations, but also by appearance of new long-period ones. Furthermore, the traces for different angles are only weakly correlated, i.e., it is not possible to scale the $B$ axis by any factor in order to reproduce the $90^{\circ}$-fluctuation pattern. This is a consequence of the 3D character of the diffusive motion of the electrons and in marked contrast to the results for 2D electron gases [10]. We checked the reproducibility of the magnetoconductance curves at the lower and the upper end of the $\theta$ range in order to verify that the samples have not changed microscopically while tilting them; cf. double traces for $90^{\circ}$ and $-10^{\circ}$ in Fig. 1. For the numerical evaluation of $B_{c}$, the orbital contribution to the magnetoconductance is subtracted by Fourier filtering which cuts off the very long wavelengths $\Delta B>5 \mathrm{~T}$. These wavelengths correspond to areas smaller than $l^{2}$ and therefore do not contribute essentially to the quantum interference of diffusive electron paths which are of interest in this paper.

The resulting values for $B_{c}(\theta)$ normalized to the values at $\theta=90^{\circ}\left(B_{c \perp}\right)$ for the five samples are shown in Fig. 2. The data for each sample are offset by four along the field axis [14]. Contrary to what one might expect at first sight, $B_{c}$ is not determined simply by $A_{\perp}$. For magnetic-field orientation along the axis of the sample, $A_{\perp}=w d$, and one would naively expect $B_{c}(0) / B_{c \perp}$ to be independent of $w$ since $B_{c \perp} \sim(L w)^{-1}[12,13]$. The difference with our data cannot be attributed to contact pads as mentioned above. Rather, the effective surface area is in fact influenced by the sample length $L$ because the projection of a typical diffusive electron trajectory perpendicular to the magnetic field will wind around the magneticfield axis many times, each time enclosing an area of order $w d$. The number $N$ of windings obviously depends on the length $L$ of the sample and may be estimated

TABLE. I. Sample number, length $L(\mathrm{~nm})$, width $w(\mathrm{~nm})$, thickness $d(\mathrm{~nm})$, elastic mean free path $l(\mathrm{~nm})$, amplitude of magnetoconductance fluctuations $\mathrm{rms} G(B)\left(e^{2} / h\right)$ with statistical error, correlation field at normal direction $B_{c \perp}(\mathrm{mT})$, normalized maximum correlation field $B_{c}(0) / B_{c \perp}$ at $\theta=0$, and rms $G(\theta)\left(e^{2} / h\right)$ of conductance fluctuations as a function of $\theta$ for the samples 1 to 5 .

\begin{tabular}{rrrrccrrc}
\hline \hline & \multicolumn{10}{c}{$\mathrm{rms}$} \\
No. & \multicolumn{1}{c}{$L$} & $w$ & $d$ & $l$ & $G(B)$ & $B_{c \perp}$ & $B_{c}(0) / B_{c \perp}$ & $G(\theta)$ \\
\hline 1 & 500 & 45 & 25 & 26 & $0.26 \pm 0.03$ & 112 & 3.0 & \\
2 & 500 & 65 & 27 & 30 & $0.24 \pm 0.03$ & 80 & 5.3 & \\
3 & 500 & 120 & 25 & 28 & $0.30 \pm 0.04$ & 35 & 7.4 & 0.32 \\
4 & 1000 & 140 & 27 & 30 & $0.20 \pm 0.03$ & 39 & 10.5 & \\
5 & 1000 & 360 & 25 & 28 & $0.33 \pm 0.04$ & 20 & 11.9 & 0.29 \\
\hline \hline
\end{tabular}




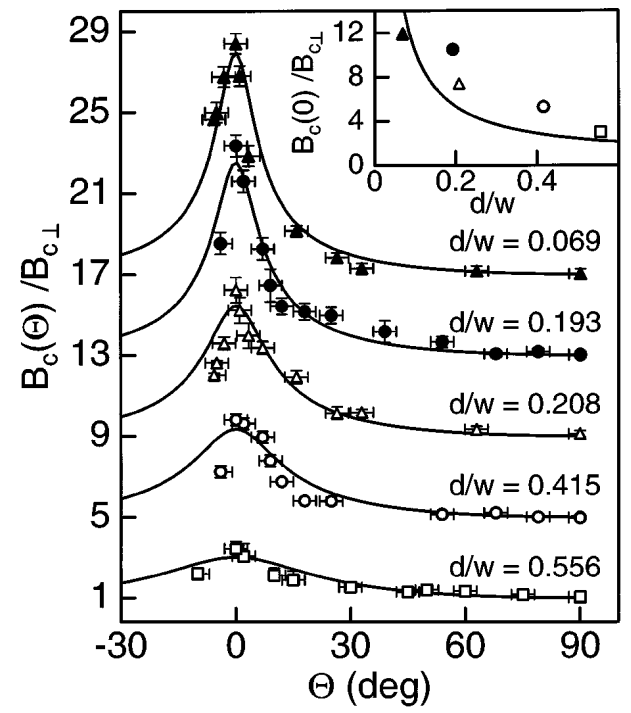

FIG. 2. Normalized correlation field $B_{c} / B_{c \perp}$ as a function of the angle $\theta$ between magnetic field and current direction for samples 1 to 5 , each offset for clarity by 4 . Error bars include numerical accuracy of $B_{c}$ obtained from the HWHM of $F(\Delta B)$. The solid lines represent the expected behavior according to Eq. (5) (see text). Inset: Fitted maximum values of $B_{c}(0) / B_{c \perp}$ as a function of $d / w$.

as follows. The average time it takes for the particle to diffuse the full width of the sample is $t_{w} \simeq w^{2} / D$, whereas the time required to diffuse from lead to lead over the length $L$ of the sample is $t_{L} \simeq L^{2} / D$. The resulting number of windings is $N \sim t_{L} / t_{w} \sim L^{2} / w^{2}$. Since the windings fluctuate between positive and negative sense of orientation, the net result of adding the contributions of all windings along the length of the sample is expressed by the effective area $A_{\phi} \simeq w d \sqrt{N} \simeq L d$, i.e., independent of the width. Hence $B_{c}(0) / B_{c \perp} \sim w / d$.

A more quantitative calculation can be done along the lines of the treatment by Lee, Stone, and Fukuyama [7]. The correlation function $F(\Delta B)=\langle G(B) G(B+\Delta B)\rangle$ is determined by eigenvalues $\lambda_{m}$ of the diffusion operator in the magnetic field $\Delta B$,

$$
D(-i \nabla-e \Delta A)^{2} Q_{m}(r)=\lambda_{m} Q_{m}(r), \quad \operatorname{rot} \Delta A=\Delta B,
$$

subject to the boundary condition $Q_{m}=0\left(\nabla_{n} Q_{m}=0\right)$ at the boundary with perfect leads (or vacuum, respectively). Choosing the coordinate system in such a way that $L\|z, w\| x$, and $d \| y$, these eigenvalues for $\Delta B=0$ are given by $\lambda_{m}=D(\pi / L)^{2} \tilde{\lambda}_{m}$,

$$
\tilde{\lambda}_{m_{z}, m_{x}, m_{y}}=m_{z}^{2}+m_{x}^{2}\left(L^{2} / w^{2}\right)+m_{y}^{2}\left(L^{2} / d^{2}\right),
$$

where $m_{z}=1,2, \cdots$ and $m_{x, y}=0,1,2, \cdots$. In the quasi1D geometry $L \gg w, d$, only $m_{x}=m_{y}=0$ eigenvalues contribute essentially to the correlation function $F(\Delta B) \propto$ $\sum_{m_{x}, m_{y}, m_{z}} \tilde{\lambda}_{m_{z}, m_{x}, m_{y}}^{-2}$.

Moreover, the sum is dominated by the few lowest values of $m_{z}$, and can be well approximated by the contribution of the lowest eigenvalue $\tilde{\lambda}_{1,0,0}=1$. To estimate the correlation field $B_{c}$, one can evaluate the perturbative correction $\delta \tilde{\lambda}_{1,0,0}(\Delta B)=\tilde{\lambda}_{1,0,0}(\Delta B)-1$ and require that $\delta \tilde{\lambda}_{1,0,0}\left(B_{c}\right)=1$. For the magnetic field lying in the $(L, d)$ plane and forming an angle $\theta$ with the $L$ axis we find

$$
\begin{aligned}
\delta \tilde{\lambda}_{1,0,0}(\Delta B)= & \frac{1}{3}\left(\frac{\Delta B L w}{\phi_{0}} \sin \theta\right)^{2} \\
& +\frac{1}{3}\left(\frac{\Delta B L d}{\phi_{0}} \cos \theta\right)^{2} \beta(w / d),
\end{aligned}
$$

where the function $\beta(z)$ is given by

$$
\beta(z)=1-\frac{768}{\pi^{6}} \sum_{m_{x}, m_{y}=1,3, \cdots} \frac{1}{m_{y}^{4}\left(m_{x}^{2}+z^{2} m_{y}^{2}\right)} .
$$

For large values of the aspect ratio $z=w / d \gg 1$ the function $\beta(z)$ behaves as $\beta(z) \simeq 1-0.63 z^{-1}$, and this formula can be used as a reasonable approximation in the whole region $z>1$.

The correlation field $B_{c}$ can therefore be estimated as

$B_{c}(\theta)=\frac{B_{c \perp}}{\sqrt{\sin ^{2} \theta+\alpha \cos ^{2} \theta}}, \quad \alpha=\left(\frac{d}{w}\right)^{2} \beta(w / d)$,

where $B_{c \perp}=\sqrt{3} \phi_{0} /(L w)$ is the correlation field for the normal orientation $\theta=90^{\circ}$. In particular, for $\theta=0^{\circ}$ we find $B_{c}(0) \sim \phi_{0} / L d$, in full agreement with the arguments based on the path representation and presented above.

The solid lines in Fig. 2 represent fits according to Eq. (5) with $\alpha$ considered as fitting parameter. The inset shows the fitted values of $\alpha^{-1 / 2}=B_{c}(0) / B_{c \perp}$ vs $d / w$ together with the theoretically expected dependence (solid line). We find good agreement between experimental results and fits.

Some of the remaining discrepancies can probably be attributed to a limited accuracy of the definition of the correlation field $B_{c}$. Experimentally, it is obtained from the HWHM of $F(\Delta B)$, whereas theoretically it is estimated from the leading order $\left[\sim(\Delta B)^{2}\right]$ perturbative correction to $F(\Delta B)$. A more accurate comparison would require evaluation of the whole function $F(\Delta B)$ at different values of $\theta$, which cannot be done analytically. Note also that our samples are on the borderline of the validity of the 3D diffusion theory, since $d \approx l$ and for the narrowest samples $w \sim l$. For completeness we should mention that usually the quasiballistic character $w<l$ is invoked to explain a reduced fluctuation amplitude [4,5], which we do not observe here.

We performed additional measurements of $G(\theta)$ with constant $B=4 \mathrm{~T}$ on samples 3 and 5 for the angular range from $0^{\circ}$ to $27^{\circ}$. The results for sample 3 are plotted in Fig. 3. The two traces for sweeping towards increasing angles (upper curve) and decreasing angles (lower curve) are highly reproducible, but some hysteretic behavior due to nonlinearities in the motion of the mechanical setup 


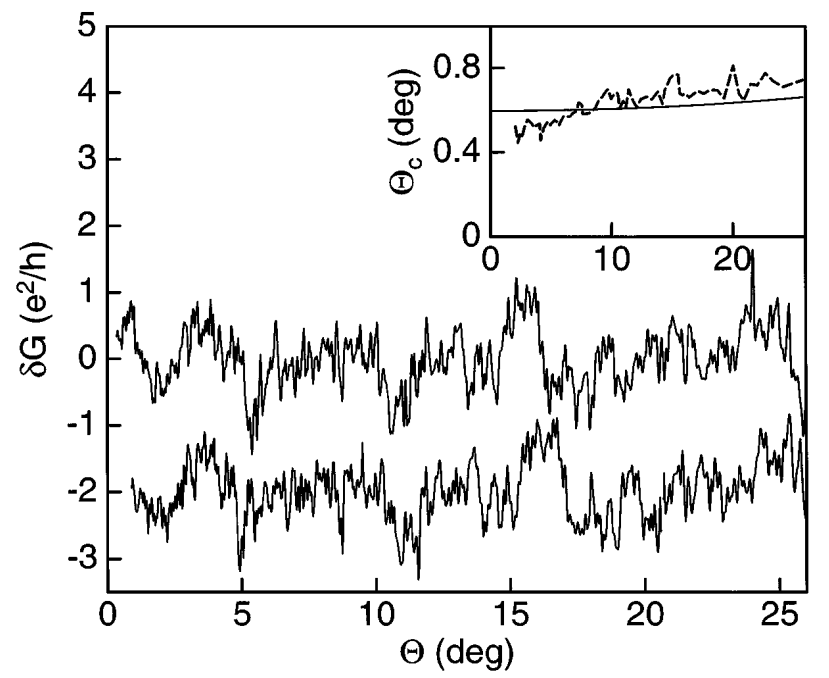

FIG. 3. Conductance fluctuations of sample 3 as a function of angle $\theta$. Upper (lower) curve: Fluctuations recorded with increasing (decreasing) $\theta$. Inset: Correlation angle $\theta_{c}$ vs $\theta$. The solid line represents the expected behavior according to Eq. (7) scaled by a factor of 0.35 .

is present. The maximum angular shift between "up" and "down" sweeps of about $1.5^{\circ}$ reflects the accuracy of the angle adjustment. The rms amplitude $0.32 e^{2} / h$, deduced from the cross correlation of both traces, is in accordance with the results of the magnetoconductance curves. In analogy to the correlation field $B_{c}$ we define a correlation angle $\theta_{c}$ which describes the typical angular shift necessary to produce a fluctuation $\Delta G \approx e^{2} / h$. Changing the angle $\theta$ by $\theta_{c}$ produces a flux change of $\phi_{0}$ through areas surrounded by "typical" trajectories. Obviously $\theta_{c}$ is not constant for the whole angular range, but increases with $\theta$. Therefore we calculate $\theta_{c}$ as a function of $\theta$ for angular ranges including about 25 fluctuations of $G(\theta)$. The result is plotted in the inset of Fig. 3. Because of the reduced $\theta$ range, the scatter is rather large. We observe a similar behavior for sample 5 where $\theta_{c}$ increases from about $0.15^{\circ}$ at $\theta=0$ to $0.3^{\circ}$ at $\theta=27^{\circ}$.

One can describe the angular correlations $\langle G(\theta) G(\theta+$ $\Delta \theta)\rangle$ at a fixed amplitude of the magnetic field $B$ in the same way as the magnetoconductance curves. We find

$$
\delta \tilde{\lambda}_{1,0,0}(\Delta \theta)=\frac{1}{3}\left(\frac{B L w}{\phi_{0}} \Delta \theta\right)^{2}\left[\cos ^{2} \theta+\alpha \sin ^{2} \theta\right]
$$

and, consequently, the correlation angle $\theta_{c}$ is estimated as

$$
\theta_{c}=\sqrt{3} \frac{\phi_{0}}{B L w} \frac{1}{\sqrt{\cos ^{2} \theta+\alpha \sin ^{2} \theta}},
$$

with $\alpha$ as defined in Eq. (5). This functional behavior is plotted in the inset of Fig. 3 using the value of $\alpha$ determined from the fit of $B_{c}(\theta)$. The absolute values of $\theta_{c}$ for all angles are scaled by a factor of 0.35 [15]. Although the weak increase of $\theta_{c}$ [Eq. (7)] is in qualitative agreement with the experimental data, a more thorough analysis would require an extension of the measurements to higher angles.

In conclusion, we have demonstrated for the first time the angular dependence of UCF in diffusive metal wires by measuring both the magnetoconductance for different angles between current and field directions, and "angular conductance" in a constant field. Extension of the established theory of UCF [7] to the case of 3D diffusive motion of electrons describes the observations with reasonable accuracy.

We would like to thank A. Sypli and H. Weber for their help in sample preparation, C. Paschke, C. Obermair, and R. Häussler for performing part of the measurements. We are indebted to R. Schäfer, K. Hecker, H. Hegger, and N. Birge for helpful discussions. This work was performed within the research program of SFB 195 supported by the Deutsche Forschungsgemeinschaft.

*On leave from St. Petersburg Nuclear Physics Institute, 188350 Gatchina, St. Petersburg, Russia.

[1] C. P. Umbach et al., Phys. Rev. B 30, 4048 (1984).

[2] R. A. Webb et al., Phys. Rev. Lett. 54, 2696 (1985).

[3] A. B. Fowler et al., Phys. Rev. Lett. 48, 196 (1982).

[4] U. Murek et al., Phys. Rev. Lett. 70, 841 (1993).

[5] P. A. M. Holweg et al., Phys. Rev. Lett. 67, 2549 (1991); Phys. Rev. B 48, 2479 (1993).

[6] D. Mailly and M. Sanquer, in Quantum Coherence in Mesoscopic Systems, edited by B. Kramer (Plenum Press, New York, 1991), pp. 401ff.

[7] P. A. Lee and A. D. Stone, Phys. Rev. Lett. 55, 1622 (1985); P. A. Lee et al., Phys. Rev. B 35, 1039 (1987).

[8] B. L. Altshuler, Pis'ma Zh. Eksp. Teor. Fiz. 41, 530 (1985) [JETP Lett. 41, 648 (1985)].

[9] For the definition of the UCF dimensionality, see Ref. [7].

[10] S. B. Kaplan and A. Hartstein, Phys. Rev. Lett. 56, 2403 (1986); R. P. Taylor et al., Surf. Sci. 196, 52 (1988).

[11] In the quasi-1D case the length scale $l_{T}=\left(h D / k_{B} T\right)^{1 / 2}$ does not affect the determination of $B_{c}$ and is therefore neglected in the discussion presented here.

[12] E. Scheer et al., J. Vac. Sci. Technol. B 12, 3171 (1994).

[13] E. Scheer et al., Physica (Amsterdam) 218B, 85 (1996).

[14] In [13] the $B_{c}(\theta)$ dependence of a further sample with $L=500 \mathrm{~nm}, w=100 \mathrm{~nm}$, and $d=25 \mathrm{~nm}$ was found to coincide with the behavior of sample 1 . In the light of the present systematic experiments this is due to a measuring error because of the smaller field range employed for that particular sample. The $B_{c \perp}$ values of all samples obtained for $\theta=90^{\circ}$ are in good agreement with the geometrical $B_{c}(w)$ dependence investigated in [13].

[15] Our calculation procedure allows one to determine $\theta_{c}$ up to an overall numerical factor of order of unity. 